\title{
Liquid biopsies for the diagnosis and surveillance of primary pediatric central nervous system tumors: a review for practicing neurosurgeons
}

\author{
Michael T. Bounajem, BS, ${ }^{1}$ Michael Karsy, MD, PhD, MSc, ${ }^{2}$ and Randy L. Jensen, MD, PhD² \\ 'Long School of Medicine, University of Texas Health, San Antonio, Texas; and 'Department of Neurosurgery, Clinical \\ Neurosciences Center, University of Utah, Salt Lake City, Utah
}

OBJECTIVE Primary brain tumors are the most common cause of cancer-related deaths in children and pose difficult questions for the treating physician regarding issues such as the risk/benefit of performing a biopsy, the accuracy of monitoring methods, and the availability of prognostic indicators. It has been recently shown that tumor-specific DNA and proteins can be successfully isolated in liquid biopsies, and it may be possible to exploit this potential as a particularly useful tool for the clinician in addressing these issues.

METHODS A review of the current literature was conducted by searching PubMed and Scopus. MeSH terms for the search included "liquid biopsy," "brain," "tumor," and "pediatrics" in all fields. Articles were reviewed to identify the type of brain tumor involved, the method of tumor DNA/protein analysis, and the potential clinical utility. All articles involving primary studies of pediatric brain tumors were included, but reviews were excluded.

RESULTS The successful isolation of circulating tumor DNA (ctDNA), extracellular vesicles, and tumor-specific proteins from liquid biopsies has been consistently demonstrated. This most commonly occurs through CSF analysis, but it has also been successfully demonstrated using plasma and urine samples. Tumor-related gene mutations and alterations in protein expression are identifiable and, in some cases, have been correlated to specific neoplasms. The quantity of ctDNA isolated also appears to have a direct relationship with tumor progression and response to treatment.

CONCLUSIONS The use of liquid biopsies for the diagnosis and monitoring of primary pediatric brain tumors is a foreseeable possibility, as the requisite developmental steps have largely been demonstrated. Increasingly advanced molecular methods are being developed to improve the identification of tumor subtypes and tumor grades, and they may offer a method for monitoring treatment response. These minimally invasive markers will likely be used in the clinical treatment of pediatric brain tumors in the future.

https://thejns.org/doi/abs/10.3171/2019.9.FOCUS19712

KEYWORDS liquid biopsy; pediatric; brain tumor; medulloblastoma; diffuse intrinsic pontine glioma; neurooncology

$\mathrm{P}$ RIMARY brain tumors are pervasive in pediatric neurosurgery, with an estimated prevalence of 35.4 per 100,000 and an incidence of 4.9 per 100,000 per year. ${ }^{15}$ Additionally, primary brain tumors are the most common cause of cancer-related deaths in children, with malignant neoplasms comprising up to $71 \%$ of all pediatric primary brain tumors. ${ }^{12,15}$ The treatment of said tumors depends on a combination of tumor type and tumor location relative to resectability. For example, although some tumors, such as those in the pineal region, pose great surgical challenges, they are highly sensitive to chemotherapy and radiotherapy (germinomas, in particular) and are therefore treated in that fashion. ${ }^{1}$ Other tumors, such as supratentorial benign astrocytomas, are typically eas- ily accessible and do exceedingly well with resection. On the other end of the spectrum, tumors such as diffuse intrinsic pontine gliomas (DIPGs) are not always amenable to resection because of the risk related to their brainstem location, which, combined with their significant resistance to treatment, means that children with DIPGs generally have a dismal prognosis. Even stereotactic biopsy for the diagnosis of DIPG is not without potential risk; therefore, the true benefit of doing so must be carefully considered.

The indirect assessment of tumors via analysis of corporeal fluids, otherwise known as the liquid biopsy, has recently gained significant favor in many aspects of oncological diagnosis and surveillance (Fig. 1)..$^{10,14,23}$ Common sources for liquid biopsies include the CSF, urine,

ABBREVIATIONS $c f D N A=$ cell-free DNA; ctDNA = circulating tumor DNA; ddPCR = diffuse-droplet PCR; DIPG = diffuse intrinsic pontine glioma; $E V$ = extracellular vesicle; $\mathrm{GBM}=$ glioblastoma; $\mathrm{MB}=$ medulloblastoma; $\mathrm{PCR}$ = polymerase chain reaction; $\mathrm{PGD} 2 \mathrm{~S}$ = prostaglandin $\mathrm{D} 2$ synthase .

SUBMITTED August 31, 2019. ACCEPTED September 24, 2019.

INCLUDE WHEN CITING DOI: 10.3171/2019.9.FOCUS19712. 
and blood or blood components; these elements are collectively referred to as the liquid biome. ${ }^{3}$ Liquid biopsies exploit the fact that tumors, particularly quickly growing tumors, shed biological material that is carried to satellite locations in the body. ${ }^{11}$ This provides an easily accessible and often less-invasive avenue for the sampling of tumor material, which has been successfully used to identify genetic predictors of prognosis and treatment response in adult glioblastoma (GBM). ${ }^{2}$ Many components of neoplastic cells have been identified within the liquid biome, including circulating tumor DNA (ctDNA), circulating RNA, extracellular vesicles (EVs), micro-RNAs, proteins, and even individual circulating tumor cells. ${ }^{3}$ Liquid biopsies have been widely studied in a variety of high-grade pediatric lesions, mainly neuroblastoma, Wilms tumor, and sarcoma, as well as in a number of adult brain tumors, including GBM. Herein lies the possible utility of liquid biopsies in pediatric brain tumors: the ability to safely identify and monitor a child's tumor. In doing so, the surgeon and oncologist may have a greater capability of evaluating a patient's treatment response, diagnosing lesions at earlier stages, evaluating leptomeningeal spread, performing serial surveillance, evaluating a complete tumor volume rather than areas only on needle biopsy area, and identifying molecular markers for adjuvant treatment. The objective of this review is to characterize the current state and utility of liquid biopsies in pediatric primary brain tumors.

\section{Methods}

A literature review was conducted to examine the extant studies on liquid biopsies, with particular attention paid to the options for indirectly isolating tumor samples, the success in doing so in vivo and in vitro, clinical applications, diagnosis, and the monitoring of changes over time. A PubMed and Scopus search for MeSH terms including "liquid biopsy," "brain," "tumor," and "pediatrics" in all fields was performed. Although articles were not excluded or included based on type of pediatric CNS tumor studied, the proportion of studies on DIPGs and medulloblastomas (MBs) is larger than other tumor types. Reviews were excluded from analysis.

\section{ctDNA}

Of the many possible analyzable targets for liquid biopsies in pediatric brain tumors, ctDNA is perhaps the most commonly studied. The origin of ctDNA lies with cell-free DNA (cfDNA), which consists of small DNA fragments that are released by dying cells, including both normal and cancerous cells. ${ }^{25}$ By nature, cfDNA is therefore nonspecific, accounting for $0.1 \%-10 \%$ of total cfDNA, and represents a catch-all of the many cell lines undergoing even moderate turnover. In patients with cancers such as aggressive brain tumors, this means that a greater portion of cfDNA is produced by dying tumor cells potentially being detectable. ctDNA also shows a short half-life (approximately 1-2 hours) in the blood stream, suggesting the possibility that changes can be detected after treatments. This fraction is known as ctDNA and can be used to identify characteristics of the tumor without ever touching the tumor itself. ${ }^{8}$ ctDNA was previously isolated using either Sanger sequencing or reverse transcription polymerase chain reaction (PCR), but investigators have recently found that diffuse-droplet PCR (ddPCR) is much more sensitive than previously employed methods.,26 Although its application in the study of pediatric brain tumors is relatively new, ctDNA has already successfully been used to monitor response to therapy in cancers such as those of the colon and breast, as well as melanoma..$^{922,23}$ This function may be of particular importance considering that the current methods for monitoring response to therapy (imaging studies) are likely less accurate than previously believed because of a phenomenon known as pseudoprogression. ${ }^{4}$

For ctDNA to be of any use in the diagnosis and monitoring of pediatric brain tumors (or any other tumor, for that matter), it must first be proven to be isolatable from a readily accessible source (Table 1 ). The methods for sample collection differ slightly depending on body location (e.g., CSF, serum) and the laboratory, but general, good laboratory practices are applicable. Blood samples may be collected in a potassium ethylenediaminetetraacetic acid tube and centrifuged for the isolation of plasma. CSF may also be centrifuged to rid the sample of any accidentally collected debris and flash frozen at $-80^{\circ} \mathrm{C}$. Stallard et al. ${ }^{20}$ have shown that ctDNA, specifically copy numbers of the H3K27 mutation, could be isolated from DIPG cells in vitro through the use of ddPCR. In their study, DIPG007 cells were cocultured with normal human astrocytes, and samples from the culture media were intermittently harvested and analyzed. Perhaps more significantly, they also showed a direct relationship between the rate of cell proliferation and quantity of ctDNA isolated, whereby more rapidly proliferating cell cultures produced more ctDNA. An in vivo correlate has been demonstrated by Wang et al., ${ }^{27}$ who found that higher-grade tumors were more likely to have identifiable CSF ctDNA compared with low-grade tumors, especially when near the ventricles. In their series of 35 patients, only 3 of 6 WHO grade I tumors exhibited isolatable ctDNA, whereas 18 of 19 grade III and IV tumors contained isolatable ctDNA. ctDNA was successfully collected from the other 5 patients with MB in the series, as well as 11 patients with GBM.

Huang et al. ${ }^{10}$ successfully isolated ctDNA from the CSF of children with DIPGs via Sanger sequencing and nested PCR with mutation-specific primers. They found that $H 3 K 27$ mutations were detectable in 4 of 5 patients with DIPGs and confirmed these findings with molecular analysis of tumor tissue itself. Importantly, they detected H3K27 mutations in ctDNA from a child with thalamic anaplastic astrocytoma, indicating the possibility that this specific mutation can be detected in multiple subtypes of diffuse midline gliomas. Pan et al. ${ }^{13}$ have since shown not only that tumor-related mutations can be found in CSF ctDNA but also that mutations can be isolated in ctDNA that is not found on tumor biopsy. However, neither diagnostic technique possesses absolute sensitivity or specificity, with some mutations being found on biopsy but not in ctDNA and vice versa. In their series of 57 patients (32 of whom had diffuse midline gliomas), tumor-specific mutations were identified in $82.5 \%$ of cases using deep sequencing PCR. Forty-seven patients also underwent 

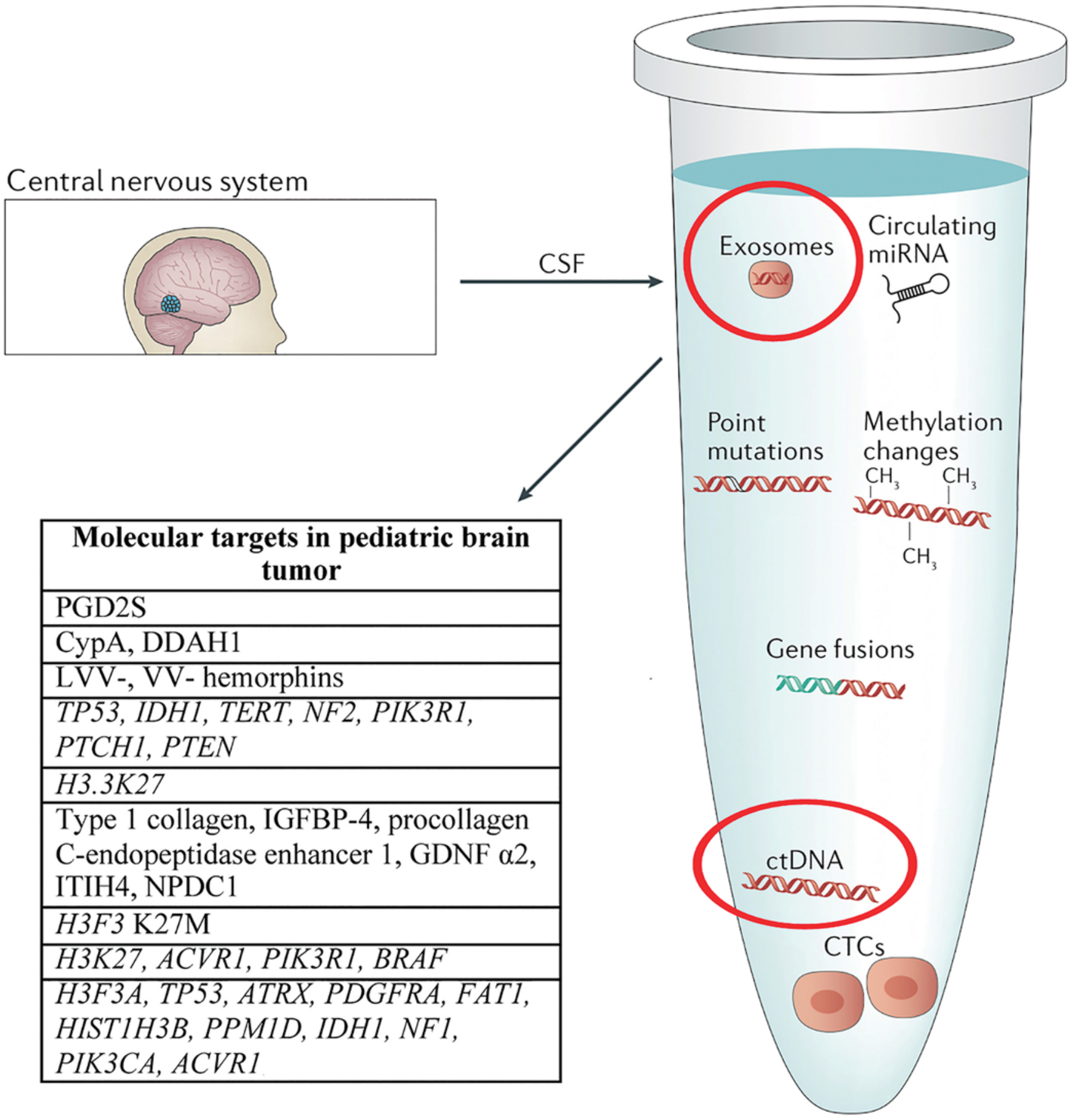

FIG. 1. Strategies to utilize liquid biopsies in disease management. Schematic representation of different body fluids (other than blood) that can contain tumor-derived molecular information-specifically, urine, CSF, saliva, and pleural effusions. The localization of the primary tumor and of any metastatic lesions influences the presence of circulating tumor-derived nucleic acids, cells, and microvesicles in individual body fluids. Point mutations, gene fusions, and methylation changes associated with the cancer can be detected through the analysis of DNA or mRNA derived from these blood-borne tumor materials. Circled in red are the two most commonly utilized targets in pediatric brain tumors-namely, ctDNA and EVs. Moreover, EVs encompass the categories of exosomes, microvesicles, and apoptotic bodies. The studied molecular targets evaluated using liquid biopsies in pediatric brain tumors are listed in Table 1. CTCs = circulating tumor cells. Adapted with permission from Macmillan Publishers Limited: Springer Nature. Nature Reviews Clinical Oncology. ${ }^{18}$ Integrating liquid biopsies into the management of cancer, by Siravegna G, Marsoni S, Siena S, Bardelli A. Copyright 2017. 


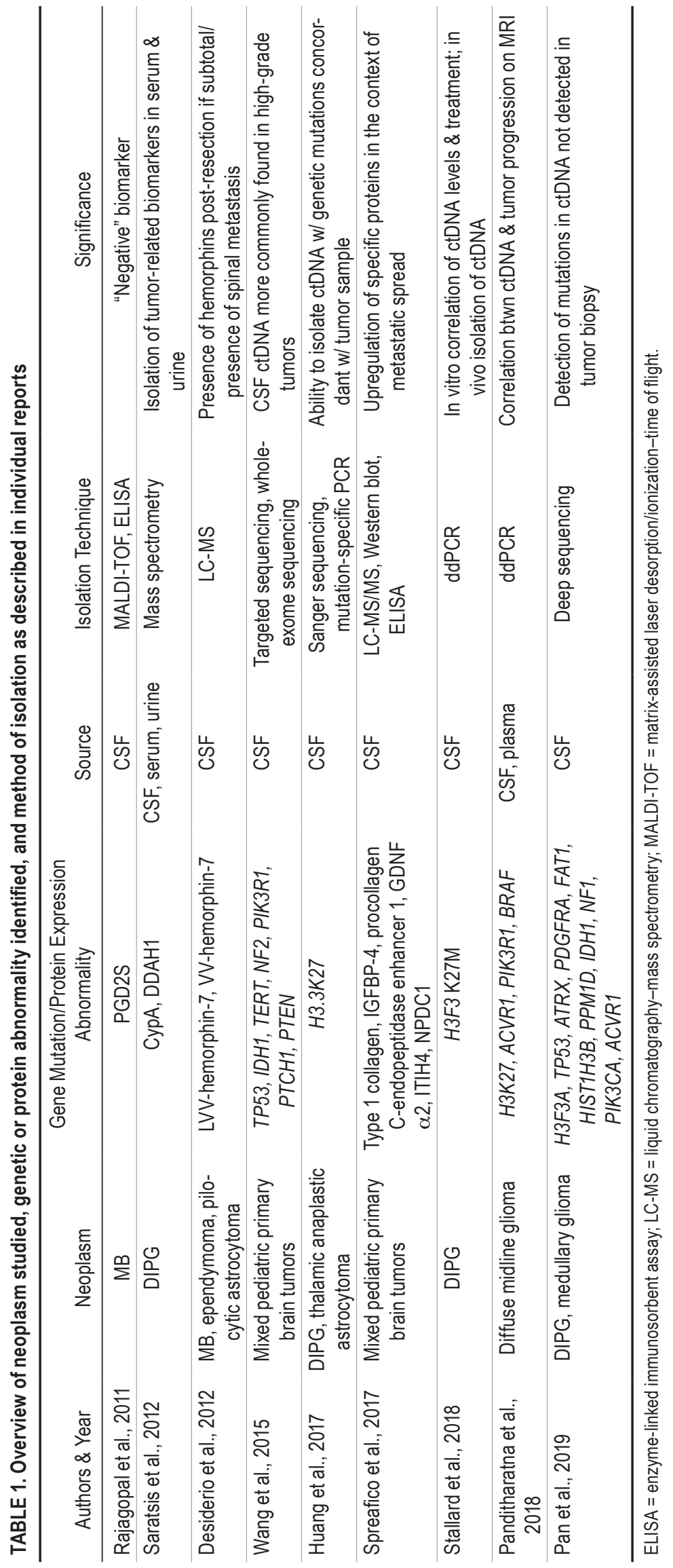


stereotactic biopsy or resection, and sequencing of DNA from the obtained tumors was conducted and the results compared with the results of CSF ctDNA analysis. Tumorspecific mutations were not identified in the samples in 10 of the biopsied cases, and $30 \%$ of the tumors lacking a mutation on biopsy examination subsequently displayed tumor-specific mutations in ctDNA isolated from the CSF. The authors also found that lower-grade tumors were associated with significantly lower detection rates of ctDNA. These findings are particularly relevant given the recent findings that conventional needle biopsies may not be sufficient for tumor assessment because of the isolated sample that is obtained. ${ }^{5}$ One of main advantages of liquid biopsies over conventional biopsies is therefore the ability to obtain a plethora of data points that represent the entire tumor. Findings that may have been missed on stereotactic biopsy may therefore be identifiable on liquid biopsy.

\section{Monitoring the Response to Treatment}

The clinical utility of liquid biopsies lies not only in the possibility of diagnosis but also in the monitoring of tumor burden and response to treatment over time. In addition to showing that ctDNA can be isolated from DIPG cells in vitro, Stallard et al..$^{20}$ demonstrated a change in ctDNA after the irradiation of tumor cells. Specifically, ctDNA containing the $H 3 K 27$ mutation increased significantly in the 72-120 hours after treatment with 8 Gy of radiation. This would support the notion that ctDNA is released as a result of tumor cell death and suggests that a direct relationship between treatment and degree of ctDNA isolation may be observable.

The most direct assessment of the use of liquid biopsies for tumor response over time was published by Panditharatna et al. in $2018 .{ }^{14}$ In this series of 48 patients with DIPGs, 110 liquid biopsies of CSF were collected and analyzed, with 22 patients undergoing serial liquid biopsies over time to assess their response to treatment. These liquid biopsies were done in parallel with serial MRI to correlate tumor size with ctDNA levels. After the patients underwent radiotherapy, ctDNA levels were found to decrease as tumor size on MRI decreased in $83 \%$ of the patients. After radiation treatment, CSF ctDNA was detected in 2 patients in whom it was not found before radiation, again suggesting an increase in ctDNA after cell damage or death. Conversely, isolation of CSF ctDNA was found to increase with disease progression, reaching the most elevated levels near death and postmortem. These findings suggest that liquid biopsies may provide a reliable method for monitoring disease progression and response to treatment in the future, although presently these findings are preliminary and require further elucidation to support the true utility of liquid biopsies.

\section{EVs and Protein Isolation}

EVs are, put simply, small blebs of plasma membrane containing DNA, RNA, proteins, and potentially any other intracellular material. ${ }^{21} \mathrm{EVs}$ are further categorized into exosomes, microvesicles, and apoptotic bodies depending on size, contents, and site of origin within the cells. They are shed by all cell types during apoptosis or in response to cell signaling, and similarly to ctDNA, they are therefore nonspecific. ${ }^{24}$ This means, however, that a portion of the EVs in the liquid biome originates from tumor cells in individuals with neoplasms. Because of the variety of cellular material contained in EVs, many different components of a tumor may be analyzed from isolating EVs. Saratsis et al. ${ }^{17}$ found through proteomic analysis that 109 proteins are upregulated in children with DIPGs. In particular, the protein folding peptide CypA and nitric oxide-regulating protein DDAH1 were significantly elevated compared with that seen in controls. Although these upregulations were found most robustly in the CSF, they were successfully isolated from urine and blood samples as well. Patterns of upregulation were also identified, suggesting that subtypes of DIPG may be identified based on proteomic analysis of patients' liquid biome.

Conversely, so-called negative biomarkers are decreased in the presence of certain neoplasms. One such example is prostaglandin D2 synthase (PGD2S), which was described by Rajagopal et al..$^{16}$ as being significantly lower in the CSF of children with MB than it was in controls. They also identified a decrease in the number of isoforms of PGD2S, suggesting that even in the absence of decreased levels, a difference may be found between children with MB and those with other tumors. Certain proteins may also be used as an avenue for monitoring a response to treatment. Desiderio et al. ${ }^{6}$ described hemoglobin chain proteins, such as LVVhemorphin-7 and VV-hemorphin-7, endogenous opioid peptides also involved in blood pressure regulation, which were present in the CSF of 13 children who harbored a variety of posterior fossa tumors. If the children underwent gross-total resection, these proteins were no longer isolatable in CSF. However, subtotal resection was found to correspond to the continued presence of these proteins in the CSF. The only other instances in which LVV-hemorphin-7 and VV-hemophorin-7 were present after resection were if drop metastases were present.

\section{Conclusions}

The management of pediatric primary brain tumors is complicated by challenges in diagnosing the disease and assessing tumor burden over multiple time points. Several discoveries described in the recent literature suggest that the use of liquid biopsies for the diagnosis and monitoring of tumors, such as DIPGs and MBs, is feasible, efficient, and minimally invasive. Liquid biopsies may also supplement current methods, such as stereotactic biopsy and MRI, because they offer an "averaged" molecular profile as a result of the multitude of the data points achieved and are not subject to the issues of pseudoprogression seen on imaging. Although the possible uses of liquid biopsies have been qualified, certain shortcomings remain. Many mutations and upregulated proteins have been found to be identifiable on liquid biopsy, but not all are specific to tumor subtypes, and therefore some overlap in diagnosis may occur. The accuracy of liquid biopsy results compared with the gold standard of tissue biopsies remains to be shown in pediatric brain tumors. Molecular techniques for liquid biopsies remain predominantly relegated to research settings. Although liquid biopsies are significantly 
less invasive compared with stereotactic biopsies, they still often warrant CSF collection, which carries risks, particularly when done in a serial fashion. In addition, it must be noted that while progress in liquid biopsies may provide physicians with an additional diagnosis/monitoring tool, overall outcomes will not necessarily be affected without a corresponding advance in treatment methods. That being said, the evidence for the clinical utility of liquid biopsies is significant and suggests a very realistic role for their use in the management of cancers such as primary pediatric brain tumors in the near future.

\section{References}

1. Abay EO II, Laws ER Jr, Grado GL, Bruckman JE, Forbes GS, Gomez MR, et al: Pineal tumors in children and adolescents. Treatment by CSF shunting and radiotherapy. J Neurosurg 55:889-895, 1981

2. Balaña C, Ramirez JL, Taron M, Roussos Y, Ariza A, Ballester R, et al: O6-methyl-guanine-DNA methyltransferase methylation in serum and tumor DNA predicts response to 1,3-bis(2-chloroethyl)-1-nitrosourea but not to temozolamide plus cisplatin in glioblastoma multiforme. Clin Cancer Res 9:1461-1468, 2003

3. Bonner ER, Bornhorst M, Packer RJ, Nazarian J: Liquid biopsy for pediatric central nervous system tumors. NPJ Precis Oncol 2:29, 2018

4. Carceller F, Fowkes LA, Khabra K, Moreno L, Saran F, Burford A, et al: Pseudoprogression in children, adolescents and young adults with non-brainstem high grade glioma and diffuse intrinsic pontine glioma. J Neurooncol 129:109-121, 2016

5. Connolly ID, Li Y, Gephart MH, Nagpal S: The "liquid biopsy": the role of circulating DNA and RNA in central nervous system tumors. Curr Neurol Neurosci Rep 16:25, 2016

6. Desiderio C, D'Angelo L, Rossetti DV, Iavarone F, Giardina B, Castagnola M, et al: Cerebrospinal fluid top-down proteomics evidenced the potential biomarker role of LVV- and VV-hemorphin-7 in posterior cranial fossa pediatric brain tumors. Proteomics 12:2158-2166, 2012

7. Diaz LA Jr, Bardelli A: Liquid biopsies: genotyping circulating tumor DNA. J Clin Oncol 32:579-586, 2014

8. Diehl F, Schmidt K, Choti MA, Romans K, Goodman S, Li $\mathrm{M}$, et al: Circulating mutant DNA to assess tumor dynamics. Nat Med 14:985-990, 2008

9. Garcia-Murillas I, Schiavon G, Weigelt B, Ng C, Hrebien S, Cutts RJ, et al: Mutation tracking in circulating tumor DNA predicts relapse in early breast cancer. Sci Transl Med 7:302ra133, 2015

10. Huang TY, Piunti A, Lulla RR, Qi J, Horbinski CM, Tomita $\mathrm{T}$, et al: Detection of histone $\mathrm{H} 3$ mutations in cerebrospinal fluid-derived tumor DNA from children with diffuse midline glioma. Acta Neuropathol Commun 5:28, 2017

11. Mansilla C, Soria E, Ramírez N: The identification and isolation of CTCs: a biological Rubik's cube. Crit Rev Oncol Hematol 126:129-134, 2018

12. Ostrom QT, Gittleman H, de Blank PM, Finlay JL, Gurney JG, McKean-Cowdin R, et al: American Brain Tumor Association adolescent and young adult primary brain and central nervous system tumors diagnosed in the United States in 2008-2012. Neuro Oncol 18 (Suppl 1):i1-i50, 2016

13. Pan C, Diplas BH, Chen X, Wu Y, Xiao X, Jiang L, et al: Molecular profiling of tumors of the brainstem by sequencing of CSF-derived circulating tumor DNA. Acta Neuropathol 137:297-306, 2019

14. Panditharatna E, Kilburn LB, Aboian MS, Kambhampati M, Gordish-Dressman H, Magge SN, et al: Clinically relevant and minimally invasive tumor surveillance of pediatric dif- fuse midline gliomas using patient-derived liquid biopsy. Clin Cancer Res 24:5850-5859, 2018

15. Porter KR, McCarthy BJ, Freels S, Kim Y, Davis FG: Prevalence estimates for primary brain tumors in the United States by age, gender, behavior, and histology. Neuro Oncol 12:520-527, 2010

16. Rajagopal MU, Hathout Y, MacDonald TJ, Kieran MW, Gururangan S, Blaney SM, et al: Proteomic profiling of cerebrospinal fluid identifies prostaglandin D2 synthase as a putative biomarker for pediatric medulloblastoma: a pediatric brain tumor consortium study. Proteomics 11:935-943, 2011

17. Saratsis AM, Yadavilli S, Magge S, Rood BR, Perez J, Hill DA, et al: Insights into pediatric diffuse intrinsic pontine glioma through proteomic analysis of cerebrospinal fluid. Neuro Oncol 14:547-560, 2012

18. Siravegna G, Marsoni S, Siena S, Bardelli A: Integrating liquid biopsies into the management of cancer. Nat Rev Clin Oncol 14:531-548, 2017

19. Spreafico F, Bongarzone I, Pizzamiglio S, Magni R, Taverna E, De Bortoli M, et al: Proteomic analysis of cerebrospinal fluid from children with central nervous system tumors identifies candidate proteins relating to tumor metastatic spread. Oncotarget 8:46177-46190, 2017

20. Stallard S, Savelieff MG, Wierzbicki K, Mullan B, Miklja Z, Bruzek A, et al: CSF H3F3A K27M circulating tumor DNA copy number quantifies tumor growth and in vitro treatment response. Acta Neuropathol Commun 6:80, 2018

21. Théry C, Ostrowski M, Segura E: Membrane vesicles as conveyors of immune responses. Nat Rev Immunol 9:581-593, 2009

22. Tie J, Wang Y, Tomasetti C, Li L, Springer S, Kinde I, et al: Circulating tumor DNA analysis detects minimal residual disease and predicts recurrence in patients with stage II colon cancer. Sci Transl Med 8:346ra92, 2016

23. Tsao SC, Weiss J, Hudson C, Christophi C, Cebon J, Behren $\mathrm{A}$, et al: Monitoring response to therapy in melanoma by quantifying circulating tumour DNA with droplet digital PCR for BRAF and NRAS mutations. Sci Rep 5:11198, 2015

24. van der Vos KE, Balaj L, Skog J, Breakefield XO: Brain tumor microvesicles: insights into intercellular communication in the nervous system. Cell Mol Neurobiol 31:949-959, 2011

25. Volik S, Alcaide M, Morin RD, Collins C: Cell-free DNA (cfDNA): Clinical significance and utility in cancer shaped by emerging technologies. Mol Cancer Res 14:898-908, 2016

26. Wang W, Song Z, Zhang Y: A comparison of ddPCR and ARMS for detecting EGFR T790M status in ctDNA from advanced NSCLC patients with acquired EGFR-TKI resistance. Cancer Med 6:154-162, 2017

27. Wang Y, Springer S, Zhang M, McMahon KW, Kinde I, Dobbyn L, et al: Detection of tumor-derived DNA in cerebrospinal fluid of patients with primary tumors of the brain and spinal cord. Proc Natl Acad Sci U S A 112:9704-9709, 2015

\section{Disclosures}

Dr. Jensen reports being a consultant for Medtronic.

\section{Author Contributions}

Conception and design: Karsy, Bounajem. Acquisition of data: Bounajem. Analysis and interpretation of data: Karsy, Bounajem. Drafting the article: Bounajem. Critically revising the article: Bounajem. Reviewed submitted version of manuscript: all authors. Approved the final version of the manuscript on behalf of all authors: Karsy. Study supervision: Karsy, Jensen.

\section{Correspondence}

Michael Karsy: Clinical Neurosciences Center, University of Utah, Salt Lake City, UT. neuropub@hsc.utah.edu. 